Stanford University School of Medicine, Stanford, CA.

Published online ahead of print at www.jco.org on August 11, 2014.

Supported by National Institutes of Health Grants No. CA 67166 and CA 88480 and by the Silicon Valley Foundation and the Sydney Frank Foundation.

Author's disclosures of potential conflicts of interest are found in the article online at www.jco.org. Author contributions are found at the end of this article.

Corresponding author: Amato J. Giaccia, PhD, Stanford University, Department of Radiation Oncology, CCSR-S, Rm 1255, 269 Campus Dr, Stanford, CA 94305-5152; e-mail: giaccia@stanford.edu.

C 2014 by American Society of Clinical Oncology

0732-183X/14/3226w-2871w/\$20.00 DOI: $10.1200 / J C O .2014 .57 .2776$

\title{
Molecular Radiobiology: The State of the Art
}

Amato J. Giaccia

$$
\begin{array}{llllllll}
\text { A } & \text { B } & \text { S } & \text { T } & \text { R } & \text { A } & \text { C } & \text { T }
\end{array}
$$

Traditional cytotoxic agents used in cancer therapy were initially discovered based on their ability to kill rapidly dividing cells. The targets of these early-generation agents were typically one or more aspects of DNA synthesis or mitosis. Thus, dose-limiting toxicities commonly associated with these agents include GI dysfunction, immunosuppression, and other consequences of injury to normal tissues in which cells are replicating under normal physiologic conditions. Although many of these agents still play an important role in cancer therapy when given concurrently with radiation therapy, the major thrust of radiobiology research in the last two decades has focused on discovering tumor-specific traits that might be exploited for more selective targeting that would enhance the efficacy of radiotherapy with less normal tissue toxicity. These newer generation molecular targeted therapies interfere with the growth of tumor cells by inhibiting genes and their protein products that are needed specifically by the tumor for survival and expansion. These agents can be complementary to radiotherapy, a spatially targeted agent. Although there have been extraordinary technical advances in radiotherapy in recent years, we are reaching the limits of improvements that radiotherapy delivery technology can bring and need different approaches. This review will highlight promising new tumor biology-based targets and other novel strategies to reduce normal tissue injury, increase tumor control, and expand the use of radiotherapy to treat widespread metastatic disease.

\section{J Clin Oncol 32:2871-2878. (C) 2014 by American Society of Clinical Oncology}

\section{INTRODUCTION}

The goal of modern cancer therapy is to apply genetic information about a tumor to guide decisions on therapy. This personalized therapy approach is a considerable deviation from traditional combinedmodality therapy, where surgery, chemotherapy, and radiation therapy are integrated in regimens tailored to the site of origin and stage of the tumor. However, outside of a few examples of targeted therapies such as imatinib, ${ }^{1}$ to date, personalized therapy that is focused on treating the genetic alterations in a tumor has not resulted in major increases in longterm survival. Although radiation therapy has been recognized as a spatially targeted therapy because it is given in a highly focused manner to a localized region of the body to eradicate tumor viability, ${ }^{2}$ it is not considered a targeted therapy by the general oncology community in the same sense as agents designed with tumor-specific genetic characteristics in mind. Throughout this review, the term molecular targeted therapy will be used with this latter definition in mind. As we expand our understanding of the complex signaling pathways and physiologic conditions that regulate tumor growth and dissemination, there is an exciting opportunity now to learn to exploit the complementary properties of radiation therapy and molecular targeted therapies to enhance their combined efficacy, spare normal tissue effects of radiation exposure, and understand how oncogenic or tumor suppressor alterations can influence radiation response.

Classical research in the radiation sciences has been concentrated on the identification and quantification of types of DNA damage induced by radiation, understanding the genes and pathways that are used to repair DNA damage, investigating the signaling pathways used to signal cell cycle arrest, developing small molecules that target tumor hypoxia, dissecting pathways induced by radiation that activate cell death, and studying the mechanisms of dose-limiting normal tissue effects. The collective radiobiology research enterprise on these topics has brought significant insight into understanding the biologic responses of tumor cells and to a lesser extent untransformed cells exposed to ionizing radiation. However, apart from the recognition that certain DNA-damaging or mitosis-disrupting chemotherapeutic agents can be combined with radiation therapy to achieve at least additive if not synergistic effects on many solid tumors, little else of this research has yet been effectively translated into the clinical practice of radiation oncology. 
Combining molecular targeted therapy with radiotherapy remains a largely uncharted domain, but there are a few established paths that could be expanded into major highways for research. Several excellent translational examples come to mind in thinking about how oncogenic changes were shown to influence therapeutic response to ionizing radiation. The first and oldest example was the demonstration that the Ras oncogene could increase the radioresistance of rodent cells. ${ }^{3-5}$ Mechanistic studies showed that the Ras oncogene requires the addition of a farnesyl group to its carboxyl terminus by the farnesyltransferase enzyme to localize in the cell membrane and be functional. ${ }^{6}$ Inhibition of farnesyltransferase activity alone results in modest tumor growth retardation in preclinical models. ${ }^{7}$ Taken together, these insights informed the design of a clinical trial with an inhibitor of Ras as a radiosensitizer. ${ }^{8}$ Among nine patients treated, three of four evaluable patients with non-small-cell lung cancer and two of three patients with head and neck squamous cell carcinoma (HNSCC) had complete responses with combined farnesyltransferase inhibitor therapy and radiotherapy. Surprisingly, there was little normal tissue toxicity of the combination therapy in this small group of patients. Unfortunately, subsequent studies have failed to sustain enthusiasm for this combined approach. ${ }^{9}$ Nevertheless, this line of investigation was an important first step toward establishing that novel agents targeting specific oncogenic changes could safely be combined with radiotherapy and were worthy of continued exploration.

A second more recent example of targeted therapy with radiation is the combination of epidermal growth factor receptor (EGFR) antibody with radiotherapy for HNSCC. ${ }^{10,11}$ The biologic rationale for these studies was based on the finding that overexpression of EGFR was correlated with tumor radioresistance. In preclinical models, treatment of tumors with EGFR inhibitors resulted in tumor growth delay and, in some cases, tumor cure. ${ }^{11}$ In a large multinational clinical trial of patients with HNSCC, patients were assigned to radiotherapy alone or radiotherapy in combination with cetuximab, a monoclonal antibody to the EGFR extracellular domain. ${ }^{12}$ This phase III study demonstrated that the combination of EGFR antibody with radiotherapy resulted in a statistically significant increase in progression-free and overall survival compared with radiotherapy alone, without an increase in grade 3 or 4 toxicities beyond those found with radiotherapy alone, except for a $4 \%$ risk of infusion reactions and a $17 \%$ risk of acneiform rash. ${ }^{12,13}$ The combination of cetuximab and radiation has been approved by the US Food and Drug Administration (FDA) for HNSCC and represents the first targeted therapeutic that has been shown to increase therapeutic efficacy with radiotherapy. ${ }^{14}$

The successes of targeted therapeutics that inhibit oncogenic alterations that drive tumor growth and radiation resistance might have been received with less enthusiasm in some circles compared with advances in linear acceleratory technology, but in fact, these steps should be heralded as the beginning of a revolution in radiation oncology. The examples discussed earlier represent proof in principle that biologic modifiers can enhance the efficacy of radiotherapy in the clinic. The conceptual framework supporting the strategic clinical implementation of combinations of radiotherapy with molecular targeted agents and antiangiogenic agents is discussed further by Morris and Harari ${ }^{15}$ in this same issue of Journal of Clinical Oncology. In the following sections of this article, the focus will be oriented more toward the current preclinical pipeline of targeted biologic response modifiers, including hypoxia-targeted therapeutics, small molecules that reprogram metabolism, small-molecule radiosensitizers, novel radioprotectors, and models that access radiation and immune system interactions.

\section{CONCEPT OF THERAPEUTIC INDEX}

Fractionation of radiation doses to spare normal tissue toxicity and kill tumor cells originally led to the concept of therapeutic index. ${ }^{16}$ The therapeutic index of anticancer therapies such as radiotherapy and chemotherapy is considered favorable if the response of the tumor tissue is greater than that of normal tissue at the same dose or concentration of drug (Fig 1). In contrast, the therapeutic index is considered unfavorable if the response of tumor tissue and normal tissue is similar for the same dose of radiation or concentration of drug (Fig 1). Thus, the concept of therapeutic index has two components - the tumor

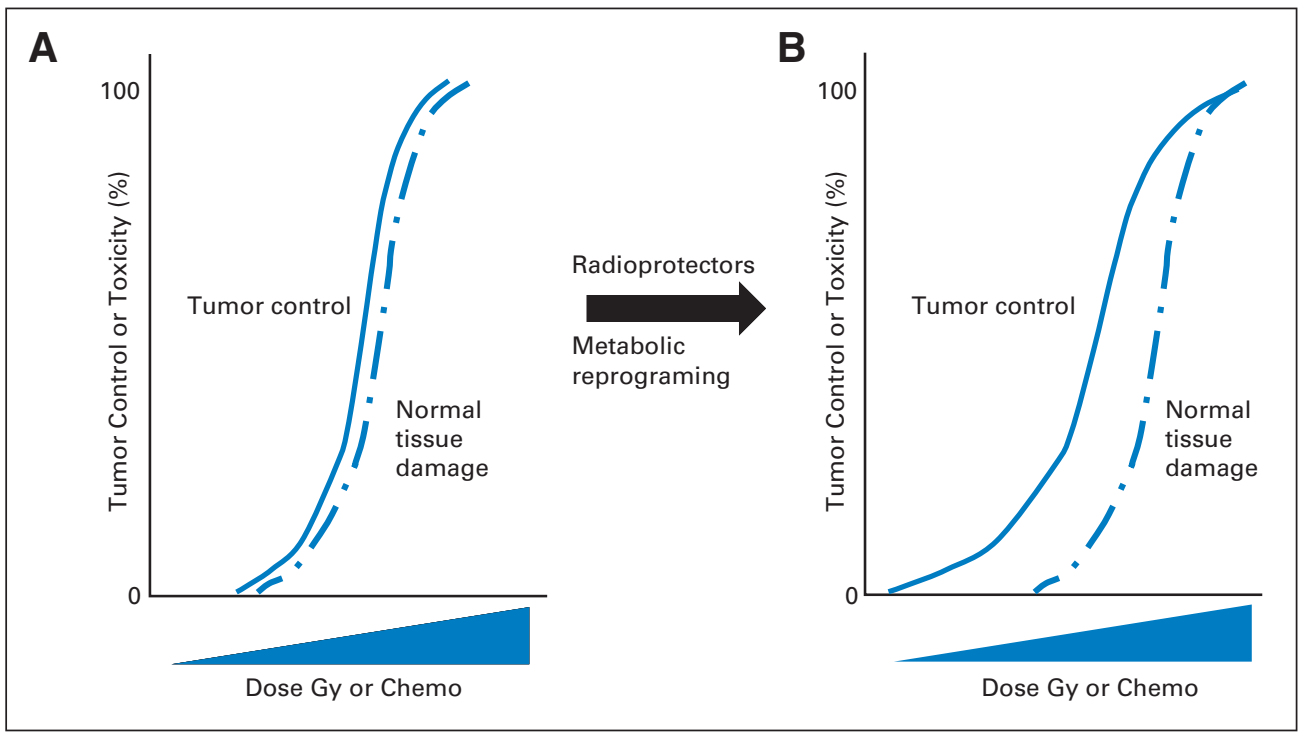

Fig 1. Dose-response relationship for tumor control and normal tissue damage: concept of therapeutic ratio. (A) An unfavorable therapeutic situation where the response of tumor and normal tissue to radiotherapy or chemotherapy is similar. (B) A favorable therapeutic index where the response of the tumor to radiotherapy or chemotherapy is significantly greater than for normal tissue. An unfavorable therapeutic index can be modified by radiosensitizing the tumor cells through metabolic reprogramming or through the radioprotection of normal tissue. Chemo, chemotherapy. 
component and the normal tissue component. However, manipulation of either part of this equation can increase the therapeutic index.

The more traditional approaches of radiosensitizing hypoxic cells with small molecules or developing hypoxia prodrugs such as tirapazamine or PR-104 that would be activated by the low-oxygen environment found in solid tumors were based on scientifically sound ideas. Unfortunately, the investigators were misled by poor preclinical model systems that overestimated the amount of tumor hypoxia that would be found in human tumors. Also, the drugs under study did not have the proper pharmacokinetic or pharmacodynamic profile to exploit tumor hypoxia in the clinic. Although the different classes of drugs that have entered clinical trials to overcome the hypoxia problem of solid tumors have yet to receive FDA approval, it should be noted that for some patients with extremely hypoxic tumors, these drugs seem to have activity. ${ }^{17,18}$ This insight leads to another reason why these drugs have not received FDA approval in that only a distinct group of patients would benefit from their use, and this patient population would need to be selected appropriately by assessing tumor hypoxia. ${ }^{19}$ It is time for change from these past approaches because they have limited impact on solid tumor response, and we should redirect our knowledge about hypoxia signaling pathways and metabolic changes that we have acquired over the last 15 years to improve solid tumor therapy. For these reasons, a departure from past approaches that did not consider normal tissue toxicity to more targeted approaches that are based on an in-depth knowledge of hypoxiaactivated signaling pathways and metabolic changes is warranted.

\section{METABOLIC REPROGRAMMING OF MITOCHONDRIAL} FUNCTION TO RADIOSENSITIZE TUMOR CELLS

Hypoxia arises because the limited supply of oxygen delivered to the tumor cannot meet the metabolic demand of the tissue. Attempts to overcome tumor hypoxia have focused primarily on the delivery of more oxygen to the tumor to make up the difference between a limited supply and the ever-increasing demand. Strategies that have been tried include increasing inspired oxygen, increasing the oxygen-carrying capacity of the blood, modulating the hemoglobin-oxygen affinity relationship, and decreasing vascular tone. All of these mechanisms are capable of physiologically increasing oxygen delivery to normal tissues. However, these approaches are significantly hindered by the limited function of the tumor vasculature. Tumor vessels are nonmuscular and contain tortuous, malformed blood vessels with blind ends and arteriovenous anastomoses. ${ }^{20}$ These architectural abnormalities limit vessel function partly by preventing laminar blood flow through a regular, branching network. The tumor has no reserve vascular capacity. These structural and functional deficiencies make it difficult, if not impossible, to deliver enough oxygen to the tumor, regardless of increased oxygen delivery to the systemic circulation and the normal organs. Theory predicts that transiently reducing oxygen consumption is a highly effective way to reduce tumor hypoxia. ${ }^{21}$ The identification of FDA-approved drugs that have a significant inhibitory effect on mitochondrial function offers the possibility of using these drugs to reduce tumor hypoxia. ${ }^{22}$ Repurposing existing drugs for metabolic radiosensitization offers an exciting possibility for rapid clinical translation and is currently an initiative through the National Center for Advancing Translational Science at the National Institutes of Health.

Attacking hypoxia by pharmacologically reducing oxygen demand is a highly innovative paradigm shift. The difficulty in imple- menting this strategy has rested in finding an agent that effectively reduces mitochondrial function without toxic adverse effects. Highdose glucose can modestly downregulate mitochondrial function in experimental tumors through the induction of the Crabtree effect. ${ }^{23} \mathrm{~A}$ bolus of glucose can shift cellular energetics to glycolysis away from oxidative phosphorylation. However, promising experimental results have not been reported in patients. One potential limitation rests in the large amount of glucose $(900 \mathrm{mg} / \mathrm{dL})$ that needs to be delivered by the inadequate tumor vasculature. As the level of glucose increases, the rate of its consumption also increases, limiting the effective dose in the tumor.

Recent published and unpublished studies have demonstrated that the biguanide metformin, approved for the treatment of type 2 diabetes, can also inhibit mitochondrial respiration, increase tumor oxygenation, and increase tumor response to radiotherapy in preclinical models. ${ }^{24-26}$ Experimental data from preclinical systems and from mathematical modeling support this concept. Interest in the combination of metformin and chemotherapy or chemoradiotherapy is also piqued by comparisons of patients with type 2 diabetes with breast and esophageal cancer who received metformin compared with patients receiving other therapy for diabetes. In one retrospective study of patients with breast cancer, the pathologic complete response was significantly higher in the cohort who received metformin and neoadjuvant chemotherapy compared with the group who did not receive metformin. ${ }^{27}$ In another retrospective study, a similar advantage in terms of higher pathologic complete response associated with metformin use was observed in patients with esophageal cancer who received neoadjuvant chemoradiotherapy. ${ }^{28}$ There remains work to be done, however, to understand how best to combine metformin with other agents to maximize the therapeutic index. For example, a recent study from Ferro et $\mathrm{al}^{29}$ has shown that diabetic patients with breast cancer treated concurrently with metformin and breast radiotherapy exhibited increased levels of locoregional toxicity and treatment breaks compared with diabetic patients receiving a different diabetic treatment. Hypoxia is known to be present in normal skin, ${ }^{30}$ and perhaps the metformin enhanced the radiation dermatitis by radiosensitizing this tissue by the same mechanism postulated to radiosensitize hypoxic tumors. The authors also discuss other mechanistic possibilities. ${ }^{29}$ In any case, a more extensive clinical evaluation of metformin and radiotherapy is warranted, especially in nondiabetic patients. It should also be noted that metformin and other related biguanides represent one therapeutic approach to increasing tumor oxygenation, and that other FDA-approved drugs that have secondary activity as inhibitors of mitochondrial activity also exist and at the least should be screened in preclinical models for potential radiosensitizing effects. Mechanistically, the effects of metformin on tumor growth inhibition and sensitization to genotoxic damage could be a result of factors other than reducing oxygen consumption rates, such as causing loss of p53 activity, ${ }^{31}$ inhibiting cancer stem cells, ${ }^{32}$ and reducing blood glucose levels. ${ }^{33}$

Because solids tumors are dependent on aerobic glycolysis for their ATP production, reducing glucose levels in tumors is a reasonable approach to radiosensitize tumors (Fig 2). Thus, a second approach to metabolic radiosensitization has been tested through targeting glucose uptake and metabolism. Studies demonstrated that 2-deoxyglucose (2-DG) could radiosensitize tumor cells by preventing glutathione recycling, ${ }^{33,34}$ thereby increasing the quantity of toxic free radicals in the irradiated cell and reducing the levels of ATP that 


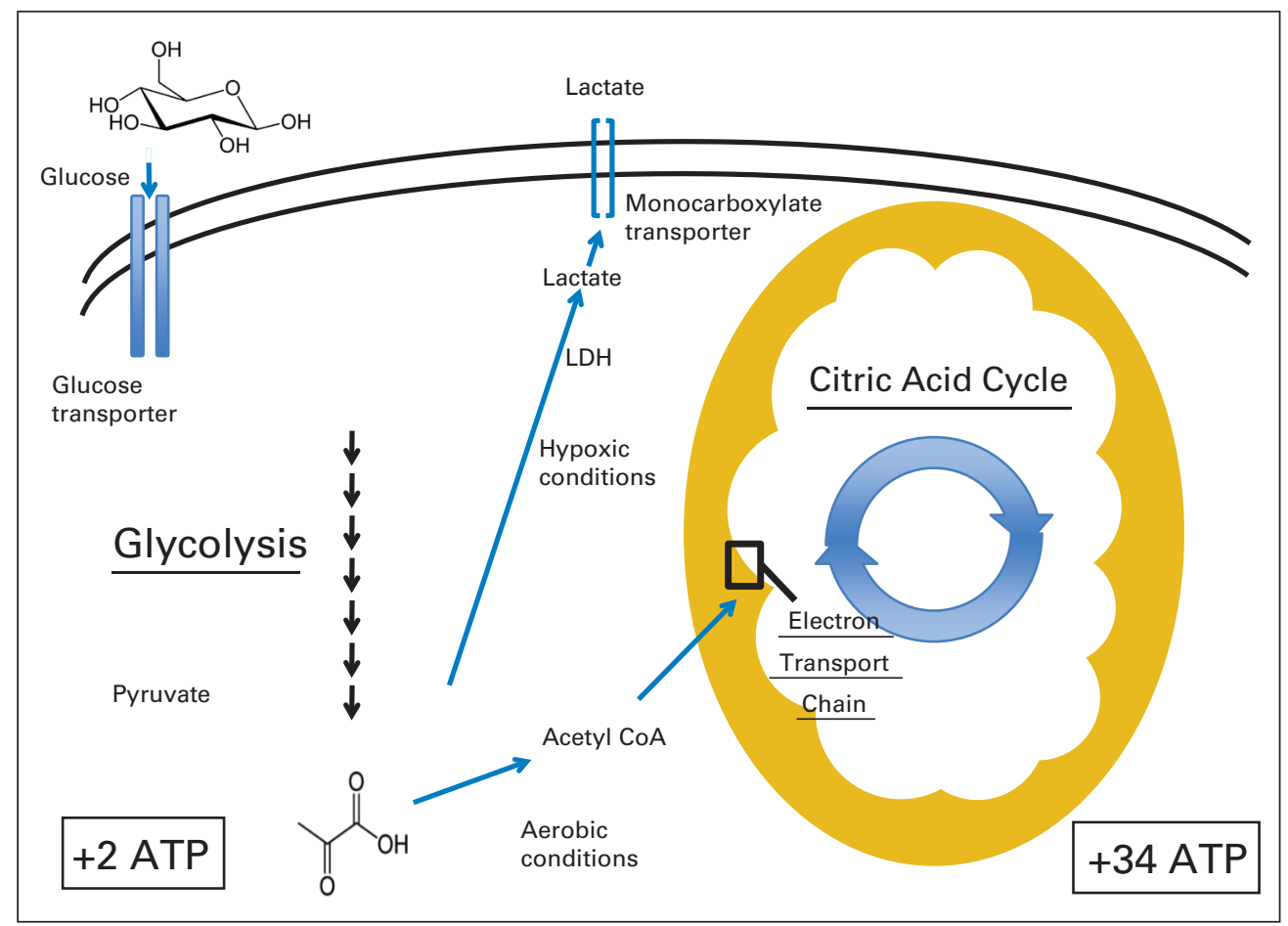

Fig 2. A simplified diagram depicts how glucose enters the cell and is metabolized. Glucose enters the cell through glucose transporters and undergoes glycolysis, which occurs under both aerobic and hypoxic conditions. For each molecule of glucose, two molecules of ATP are generated. Pyruvate, the end product of glycolysis, undergoes decarboxylation by pyruvate dehydrogenase to become acetyl-coenzyme A (acetyl CoA) and enters the citric acid cycle under aerobic conditions, which generates 34 molecules of ATP. Under hypoxic conditions, pyruvate is converted by lactate dehydrogenase (LDH) into lactate and is transported out of the hypoxic cell by monocarboxylate transporters. Aerobic cells can transport lactate into the cell and burn lactate as energy.

are needed for DNA damage signaling and repair. ${ }^{35}$ Enthusiasm for 2-DG as a radiosensitizer diminished with the observed variability in radiosensitization between cell lines. ${ }^{35}$ Part of this variability has been correlated to the functionality of the p53 tumor suppressor pathway. Studies showed that the radiosensitizing effects of 2-DG significantly increased with an intact p53 tumor suppressor pathway and diminished when 553 was inactivated. ${ }^{36}$ Interestingly, the combination of $2-D G$ and radiation was studied in the 1990s to treat patients with supratentorial high-grade glioma. ${ }^{37}$ Somewhat surprising was that all 20 patients in the trial were able to manage the combination treatment (2-DG $200 \mathrm{mg} / \mathrm{kg}+5$ Gy of whole-brain radiation weekly for 4 weeks, followed by a smaller field boost of $14 \mathrm{~Gy}$ in seven 2-Gy fractions) with no significant adverse normal tissue effects. A 2-DG dose-escalation study in patients with glioblastoma showed that the maximumtolerated dose of 2-DG that could be combined with seven onceweekly 5-Gy fractions was $250 \mathrm{mg} / \mathrm{kg}^{38} \mathrm{~A}$ randomized trial of radiotherapy with or without 2-DG has not yet been reported but would be needed to establish a benefit with the combination of 2-DG and radiation.

Mechanistically, 2-DG does not inhibit glucose uptake into the tumor cell but acts as a competitive inhibitor of glucose within the cell. During the last few years, some selective and potent glucose transport inhibitors have been developed that target different family members, especially those that are induced by changes in the tumor microenvironment such as Glut1 and Glut3 (Fig 2). ${ }^{39}$ Using more selective glucose transport inhibitors reduces normal tissue toxicity, while at the same time targeting tumor metabolism. To date, these selective small-molecule inhibitors have not been tested with radiation, but there is every expectation that they should provide the same level of sensitization as 2-DG with ionizing radiation and will kill cells with activated hypoxia-inducible factor (HIF) signaling. Furthermore, inhibiting glucose uptake in metabolism can be more readily quantified using fluorodeoxyglucose-positron emission tomography imaging than after treating tumors with 2-DG, which serves as the molecule used in fluorodeoxyglucose-positron emission tomography imaging.

Reliance on aerobic glycolysis results in increased levels of lactate, which is pumped into or out of the cell by a family of specialized transporters called monocarboxylate transporters (MCTs; Fig 2). ${ }^{40}$ Different MCTs have specialized functions in hypoxic and aerobic tumor cells. ${ }^{41}$ For example, MCT1, which is highly expressed in aerobic tumor cells, transports lactate into cells, presumably to use in oxidative metabolism to generate energy. In contrast, MCT4, which is induced by hypoxic conditions, transports lactate out of hypoxic cells to help maintain intracellular $\mathrm{pH}$ balance. Although further analysis has suggested that MCT expression and most probably function are not as simple as described earlier, MCTs represent exciting therapeutic targets to selectively kill hypoxic cells. ${ }^{42}$ For example, inhibition of MCT1 using $\alpha$-cycan-4-hydroxycinnamate (CHC) in preclinical glioblastoma models significantly reduced tumor growth and angiogenesis. ${ }^{43}$ Inhibitors of MCTs are still under investigation, but their importance in tumor metabolism makes them a prime target to consider in combination with radiotherapy.

\section{RADIOSENSITIZERS, RADIOPROTECTORS, AND STEM-CELL SALVAGE}

Despite improvements in the specificity of chemotherapeutic agents ${ }^{44}$ and the precision of external-beam radiation, ${ }^{45,46}$ many cancers only partially respond to treatments, suggesting that increasing doses of cytotoxic therapy may be needed to overcome mechanisms of resistance and to control tumor growth. Unfortunately, normal tissue adverse effects can limit the tolerance of intensive combinations of concurrent chemotherapy and radiotherapy. Over the years, many 
investigators have hoped to divine the Achilles heel of tumor radioresistance by unraveling the complexities of DNA repair. Although great progress in understanding DNA damage response and DNA strand break repair pathways has been made, to date, there has only been limited success in being able to translate this knowledge into therapeutic strategies.

For example, the ATM gene that is mutated in individuals with the radiosensitive syndrome ataxia telangiectasia has been cloned and extensively characterized. ${ }^{47}$ Screening of targeted libraries for inhibitors of ATM kinase resulted in small molecules that are relatively specific for ATM such as CP46672 and Ku55933. ${ }^{48}$ Treatment of tumor and untransformed cells with these ATM inhibitors, even for short periods of time, results in radiosensitization. Not considering the required steps in small-molecule drug development for the clinic, the conceptual problem arises of how one would develop these radiosensitizing drugs to use in combination with radiotherapy. Systemic administration of these ATM inhibitors would be problematic because of normal tissue radiosensitization located in the field of the radiation beam. The best use of these inhibitors would possibly be in the application of brachytherapy, where much of the dose will be localized to the tumor region with minimal normal tissue effects. Alternatively, they might combine safely with external-beam techniques like stereotactic body radiation therapy or charged particle therapy that achieve high-dose deposition in tumor tissue with a low integral dose accumulation in adjacent normal tissue.

An alternative approach to inhibiting DNA damage signaling or DNA repair pathways with small molecules is to use the strategy of synthetic lethality (Fig 3). ${ }^{49}$ Such an approach exploits tumor-specific mutations that are not present in normal cells by identifying either genetically or pharmacologically a target that when inhibited will

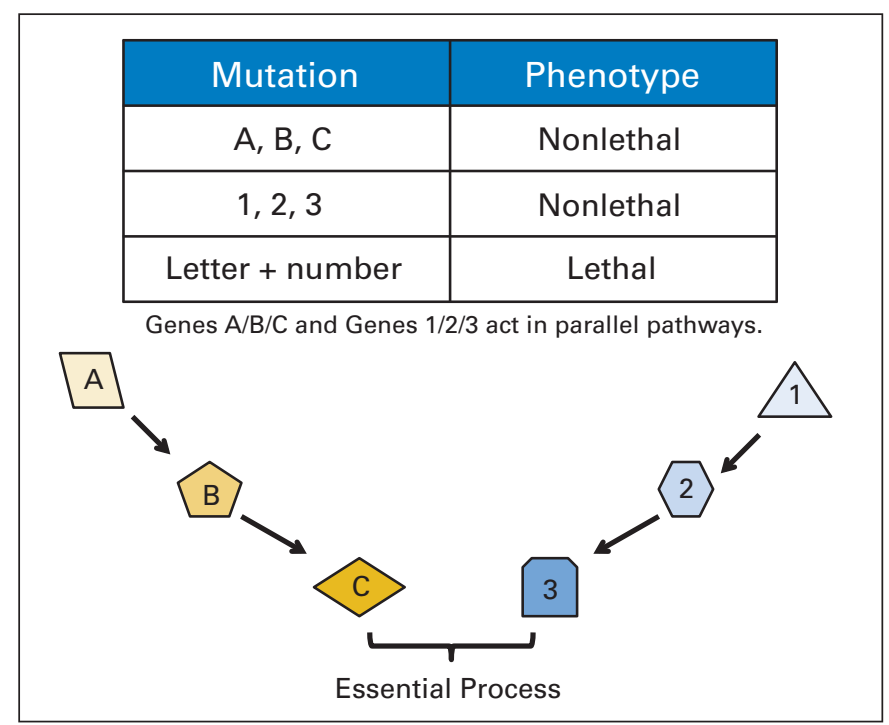

Fig 3. Synthetic lethality in cancer therapy. Traditionally, synthetic lethal interactions have been defined between two genes or pathways when their combined inactivation results in cell killing that is greater than the inactivation of each gene alone. In the example shown, inactivation of any member of the $A, B$, $\mathrm{C}$ or 1, 2, 3 pathway individually will have little effect on viability. However, when mutations in both pathways occur, cells will die. The concept of conditional synthetic lethality replaces the two-pathway concept with a pathway and a stressor such as ionizing radiation or hypoxia. In this situation, when cells would be exposed to ionizing radiation, inactivation of specific pathways would result in cell killing. cause cell death of the tumor cell and not the normal cells. The best example of synthetic lethality is the use of poly (ADP-ribose) polymerase (PARP) inhibitors with BRCA1- or BRCA2-mutated breast or ovarian cancer cells. PARP inhibition by itself has no significant cytotoxicity. However, when PARP inhibitors are applied to tumor cells with BRCA1 or BRCA2 mutations that render them deficient in homologous recombination, replication forks become stalled and collapse, leading to cell demise. ${ }^{50}$ This concept of synthetic lethality can be modified to identify conditional synthetic lethal interactions that occur when cells are exposed to external stresses such as ionizing radiation or hypoxia. This concept was nicely demonstrated by Bindra et $\mathrm{al}^{51}$ and Chan et $\mathrm{al}^{52,53}$ who showed that exposure of cells to hypoxia resulted in decreased expression of the homologous recombination protein Rad51 and sensitivity to exposure to PARP inhibitors, much like BRCA1- or BRCA2-deficient tumor cells. However, their deficiency in Rad51 and homologous recombination was based on exposure to hypoxic conditions not to genetic mutation. To date, few screens have examined synthetic lethal interactions with exposure to ionizing radiation. One elegant screen by Higgins et $\mathrm{al}^{54}$ used small interfering RNAs for 200 genes involved in DNA repair to identify the DNA polymerase POLQ as a potential tumor-specific target. They further verified that knockdown of POLQ resulted in radiosensitization of a panel of tumor cell lines and had little effect on normal tissue. These types of approaches represent a promising avenue of research for the future based on synthetic lethal interactions.

Although in theory both radiosensitization and radioprotection can increase the therapeutic index, in practice, radioprotection will possibly be easier to achieve than radiosensitization because it is difficult to get uniform radiosensitization of all tumor cells and only a small number of surviving tumor stem cells can repopulate the tumor. In contrast, only a fraction of normal cells need to be protected to regenerate or maintain function of the tissue. Even with significant technical advances in the delivery of radiotherapy, normal tissue toxicity still poses a difficult problem to address, because the only radioprotector approved to date, amifostine, has not been widely adopted because of its own adverse effects and the lack of robust normal tissue protection. ${ }^{55}$ Mechanistically, radioprotectors under consideration today fall into the following broad groups: free radical scavengers such as amifostine, ${ }^{56-58}$ inhibitors of DNA damage-induced signaling (eg, the $p 53$ tumor suppressor gene), agonists of toll-like receptor $5,{ }^{59}$ and inhibitors of the ceramide pathway. ${ }^{60}$

Although all of these approaches have merit, almost all are focused on trying to protect epithelial cells from the cytotoxic effects of DNA damage, with little attention to the underlying changes in tissue physiology induced by radiation. Recent studies by Taniguchi et $\mathrm{al}^{61}$ have demonstrated that activating pathways to promote epithelial integrity in preclinical models can increase the survival of animals that are lethally irradiated (Fig 4). As proof of principle, these investigators demonstrated that activation of the HIF-2 transcription factor in the GI tract pharmacologically through a small-molecule inhibitor of prolyl hydroxylases, a family of enzymes that hydroxylate HIF and target it for degradation under aerobic conditions, could provide long-term protection against exposure to lethal levels of totalabdominal irradiation or total-body irradiation and did not affect tumor radiosensitivity. Currently, a series of small molecules that inhibit prolyl hydroxylases, which are negative regulators of HIF-1 and HIF-2, are in clinical trials for the treatment of anemia caused by renal insufficiency. ${ }^{62}$ These molecules seem to be safe and potent in 


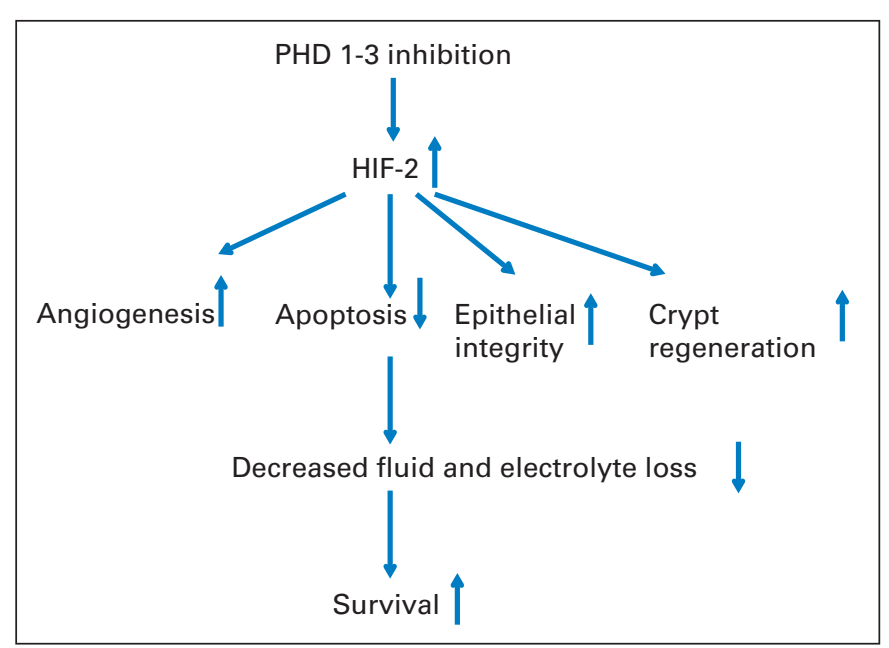

Fig 4. Protection against Gl syndrome by activation of the hypoxia-inducible factor (HIF) -2 pathway. Inhibition of prolyl hydroxylase (PHD) activity pharmacologically or genetically results in the activation of the HIF-2 pathway in the small intestine and the colon. Activation of HIF-2 in the GI tract before exposure to lethal dose of ionizing radiation increases angiogenesis, protects against apoptosis, increases epithelial integrity, and promotes crypt regeneration. The consequences of HIF-2 activation are decreased fluid and electrolyte loss and increased survival. In addition, HIF-2 can also act as a radiation mitigator when given after exposure to lethal dose of radiation.

inducing the HIF pathway and represent a potential therapeutic path to testing this concept in humans. Furthermore, the Radiation and Nuclear Countermeasures Program has been instrumental in reviving interest in the development of radioprotectors and radiation mitigators, agents that can be given after exposure to radiation to increase tissue protection. This research will bring forth new pathways and molecules that have been previously unknown and that should also be tested in the clinic.

One exciting horizon for future investigations is the use of radiotherapy to treat systemic disease. Currently, stereotactic radiotherapy may be used to treat localized tumors or a few metastases (oligometastases), and this topic is well reviewed elsewhere in this issue of Journal of Clinical Oncology. ${ }^{63}$ However, external-beam radiotherapy is not routinely used for more widespread metastatic disease because of the associated normal tissue toxicity. Let us consider the example of a patient with a primary pancreatic tumor that has metastasized to the liver. The liver is completely infiltrated; ultimately, the tumor burden will prevent the liver from functioning, and death will ensue. Unfortunately, currently available chemotherapy only inhibits the metastases in a short-term manner and never eradicates the disease completely. Radiotherapy can be highly effective in eradicating a small number of discrete tumors, but there are limitations on the use of radiotherapy when the disease is too extensive in the liver as a result of normal hepatocyte cell death, especially at the doses of radiation needed to eliminate the metastasis. For radiotherapy to be used in such a manner, we need to develop effective small-molecule radioprotectors that defend the normal hepatocytes, but not tumor tissue, from radiation-induced cell death. Such agents would revolutionize the use of radiation for the treatment of metastasis and, most importantly, might increase the long-term survival of patients with metastatic disease.
Yet another innovative strategy to overcome normal tissue toxicities is the prospect of transplanting stem cells into an organ to restore functionality after radiotherapy, a topic that has recently been reviewed extensively. ${ }^{64}$ One of the particularly intriguing models that is under study preclinically is the transplantation of healthy unirradiated hepatocytes as a means of recovery from radiation-induced liver disease (RILD). In a rodent model of RILD, intrasplenic or intraportal infusion of adult primary hepatocytes corrected RILD and improved survival of rats treated with high-dose liver radiotherapy after partial hepatectomy. ${ }^{65}$ Interestingly, the use of either single high-dose radiation or an aggressive fractionated radiotherapy regimen to a partial liver volume enhances the rate of successful engraftment of the transplanted hepatocytes. ${ }^{66}$ The clinical use of hepatocyte transplantation to correct non-RILD diseases of the liver is also an area of current investigation, and there might be a role for radiation as part of the preparative regimen in that setting. ${ }^{67}$

\section{IMMUNE CHECKPOINT BLOCKADE AND RADIOTHERAPY}

The use of radioprotectors to treat widespread systemic disease with radiation is based on traditional radiobiologic concepts. However, the use of radiation to modulate the immune system to attack metastatic disease is another revolutionary idea that is still in its infancy. ${ }^{68}$ In the last decade, a great deal of effort has been invested in understanding the antigens on tumor cells that the immune system uses to mount a response or remain dormant. In fact, the antigenic repertoire of tumor cells is in a dynamic state as a result of genomic instability, changes in cell-to-cell and cell-tomatrix contact, and exposure to the tumor microenvironment.

A major advance in immunotherapy has been brought about by the identification of inhibitory receptors on immune cells and how they respond to their ligands. These molecules act as a rheostat for the immune responses of $\mathrm{T}$ cells and have been described as T-cell checkpoints. ${ }^{69}$ These checkpoints comprise a series of costimulatory molecules such as CD28, which through ligation to CD80 or CD86 on antigen-presenting cells promote mitogenic and survival signals, or inhibitory receptors such as CTLA-4, PD-1, LAG-3, TIM-3, NKG2A, and KIRs that downregulate T-cell function when an antigen is recognized by the T-cell receptor. ${ }^{70}$ One obvious mechanism for tumors to induce tolerance is to express any of the inhibitory molecules just described. Indeed, much of the focus has been directed toward the inhibitory molecules, such as CTLA- 4 and PD-1, that can be targeted with blocking antibodies. Blocking these inhibitory molecules stimulates the $\mathrm{T}$ cell to act in an antitumor manner.

Recently, there has been great interest in combining immune checkpoint inhibitors with radiotherapy as a result of several highprofile publications describing remarkable abscopal effects with the combination of a CTLA-4 antibody, ipilimumab, and stereotactic radiation in patients with metastatic melanoma. ${ }^{71-73}$ Similarly provocative observations have been made with the combination of highdose stereotactic radiation and interleukin-2 for patients with melanoma and renal cell cancer. ${ }^{74}$ Although we still know little about the mechanisms underlying these abscopal effects, the excitement over these studies has led to a number of clinical trials that have begun or will soon begin, attempting to recapitulate the abscopal effects seen 
with melanoma and renal cell cancer, but without a thorough understanding of the underlying mechanisms behind it. ${ }^{75}$ The lack of understanding of this immune mechanism, the paucity of biomarkers except for myeloid suppressor cells, and an uncertain optimal radiation dosing schedule suggest opportunities for additional preclinical testing in animal models to fill in some of the gaps in our knowledge. Our goals should be to identify tumors that will respond to checkpoint inhibition alone versus tumors that respond to only combination therapy, to develop ways to optimize antigen stimulation through radiation exposure, and to investigate in more depth what other factors are antagonizing the immune response.

\section{SUMMARY}

The field of radiation oncology has embraced the advances in imaging and treatment delivery technology with open arms. The progress has been quite significant and has resulted in more accurate delivery of therapy and reduced normal tissue exposure. However, as we approach a plateau in the degree of benefit that can be achieved by perfecting treatment devices, improving the treatment of patients with cancer will require the field to embrace the revolution in tumor biology to develop companion therapeutics that will enhance the efficacy of radiotherapy in nontraditional ways. These new therapeutics will act as selective radiosensitizers for tumor cells, protect normal tissue from radiation damage, and modulate the immune system to its best advantage in the management of locally advanced and metastatic disease.

\section{AUTHORS' DISCLOSURES OF POTENTIAL CONFLICTS OF INTEREST}

Disclosures provided by the authors are available with this article at www.jco.org.

\section{REFERENCES}

1. Druker BJ: Translation of the Philadelphia chromosome into therapy for CML. Blood 112:4808 4817, 2008

2. Macdermed DM, Weichselbaum RR, Salama JK: A rationale for the targeted treatment of oligometastases with radiotherapy. J Surg Oncol 98:202206, 2008

3. Sklar MD: The ras oncogenes increase the intrinsic resistance of $\mathrm{NIH}$ 3T3 cells to ionizing radiation. Science 239:645-647, 1988

4. McKenna WG, Weiss MC, Endlich B, et al: Synergistic effect of the $\mathrm{v}$-myc oncogene with $\mathrm{H}$-ras on radioresistance. Cancer Res 50:97-102, 1990

5. McKenna WG, Weiss MC, Bakanauskas VJ, et al: The role of the $\mathrm{H}$-ras oncogene in radiation resistance and metastasis. Int J Radiat Oncol Biol Phys 18:849-859, 1990

6. Zhang FL, Casey PJ: Protein prenylation: Molecular mechanisms and functional consequences. Annu Rev Biochem 65:241-269, 1996

7. Kohl NE, Wilson FR, Mosser SD, et al: Protein farnesyltransferase inhibitors block the growth of ras-dependent tumors in nude mice. Proc Natl Acad Sci U S A 91:9141-9145, 1994

8. Hahn SM, Bernhard EJ, Regine W, et al: A phase I trial of the farnesyltransferase inhibitor L-778, 123 and radiotherapy for locally advanced lung and head and neck cancer. Clin Cancer Res 8:10651072, 2002

9. Haas-Kogan DA, Banerjee A, Poussaint TY, et al: Phase II trial of tipifarnib and radiation in children with newly diagnosed diffuse intrinsic pontine gliomas. Neuro Oncol 13:298-306, 2011

10. Harari PM, Huang SM: Radiation response modification following molecular inhibition of epidermal growth factor receptor signaling. Semin Radiat Oncol 11:281-289, 2001

11. Nasu S, Ang KK, Fan Z, et al: C225 antiepidermal growth factor receptor antibody enhances tumor radiocurability. Int J Radiat Oncol Biol Phys 51:474-477, 2001

12. Bonner JA, Harari PM, Giralt J, et al: Radiotherapy plus cetuximab for squamous-cell carcinoma of the head and neck. N Engl J Med 354: 567-578, 2006

13. Bonner JA, Harari PM, Giralt J, et al: Radiotherapy plus cetuximab for locoregionally advanced head and neck cancer: 5-year survival data from a phase 3 randomised trial, and relation between cetuximab-induced rash and survival. Lancet Oncol 11:21-28, 2010

14. Mehra R, Cohen RB, Burtness BA: The role of cetuximab for the treatment of squamous cell carcinoma of the head and neck. Clin Adv Hematol Oncology 6:742-750, 2008

15. Morris ZS, Harari PM: Interaction of radiation therapy with molecular targeted agents. J Clin Oncol 32:2886-2893, 2014

16. Bernier J, Hall EJ, Giaccia A: Radiation oncology: A century of achievements. Nat Rev Cancer 4:737-747, 2004

17. Rischin D, Peters LJ, O'Sullivan B, et al: Tirapazamine, cisplatin, and radiation versus cisplatin and radiation for advanced squamous cell carcinoma of the head and neck (TROG 02.02, HeadSTART): A phase III trial of the Trans-Tasman Radiation Oncology Group. J Clin Oncol 28:29892995, 2010

18. Jameson $M B$, Rischin $D$, Pegram $M$, et al: $A$ phase I trial of PR-104, a nitrogen mustard prodrug activated by both hypoxia and aldo-keto reductase $1 \mathrm{C} 3$, in patients with solid tumors. Cancer Chemother Pharmacol 65:791-801, 2010

19. Rischin D, Hicks RJ, Fisher R, et al: Prognostic significance of [18F]-misonidazole positron emission tomography-detected tumor hypoxia in patients with advanced head and neck cancer randomly assigned to chemoradiation with or without tirapazamine: A substudy of Trans-Tasman Radiation Oncology Group Study 98.02. J Clin Oncol 24:20982104, 2006

20. Vaupel P: Tumor microenvironmental physiology and its implications for radiation oncology. Semin Radiat Oncol 14:198-206, 2004

21. Secomb TW, Hsu R, Ong ET, et al: Analysis of the effects of oxygen supply and demand on hypoxic fraction in tumors. Acta Oncol 34:313-316, 1995

22. Gagnon G, Regoli D, Rioux F: Studies on the mechanism of action of various vasodilators. $\mathrm{Br} J$ Pharmacol 70:219-227, 1980

23. Snyder SA, Lanzen JL, Braun RD, et al: Simultaneous administration of glucose and hyperoxic gas achieves greater improvement in tumor oxygenation than hyperoxic gas alone. Int J Radiat Oncol Biol Phys 51:494-506, 2001
24. Zannella VE, Dal Pra $A$, Muaddi $H$, et al: Reprogramming metabolism with metformin improves tumor oxygenation and radiotherapy response. Clin Cancer Res 19:6741-6750, 2013

25. Song CW, Lee $H$, Dings RP, et al: Metformin kills and radiosensitizes cancer cells and preferentially kills cancer stem cells. Sci Rep 2:362, 2012

26. Storozhuk $Y$, Hopmans $S N$, Sanli $T$, et al: Metformin inhibits growth and enhances radiation response of non-small cell lung cancer (NSCLC) through ATM and AMPK. Br J Cancer 108:20212032, 2013

27. Jiralerspong $S$, Palla SL, Giordano $\mathrm{SH}$, et al: Metformin and pathologic complete responses to neoadjuvant chemotherapy in diabetic patients with breast cancer. J Clin Oncol 27:3297-3302, 2009

28. Skinner HD, McCurdy MR, Echeverria AE, et al: Metformin use and improved response to therapy in esophageal adenocarcinoma. Acta Oncol 52: 1002-1009, 2013

29. Ferro A, Goyal S, Kim S, et al: Evaluation of diabetic patients with breast cancer treated with metformin during adjuvant radiotherapy. Int J Breast Cancer 2013:659723, 2013

30. Bedogni B, Powell MB: Skin hypoxia: A promoting environmental factor in melanomagenesis. Cell Cycle 5:1258-1261, 2006

31. Muaddi H, Chowdhury $S$, Vellanki $R$, et al: Contributions of AMPK and p53 dependent signaling to radiation response in the presence of metformin. Radiother Oncol 108:446-450, 2013

32. Campbell RK, White JR Jr, Saulie BA: Metformin: A new oral biguanide. Clin Ther 18:360-371, 1996

33. Lin X, Zhang F, Bradbury CM, et al: 2-DeoxyD-glucose-induced cytotoxicity and radiosensitization in tumor cells is mediated via disruptions in thiol metabolism. Cancer Res 63:3413-3417, 2003

34. Coleman MC, Asbury CR, Daniels D, et al: 2-Deoxy-D-glucose causes cytotoxicity, oxidative stress, and radiosensitization in pancreatic cancer. Free Radic Biol Med 44:322-331, 2008

35. Dwarkanath BS, Zolzer F, Chandana S, et al: Heterogeneity in 2-deoxy-D-glucose-induced modifications in energetics and radiation responses of human tumor cell lines. Int J Radiat Oncol Biol Phys 50:1051-1061, 2001 
36. Sinthupibulyakit $\mathrm{C}$, Grimes KR, Domann FE, et al: p53 is an important factor for the radiosensitization effect of 2-deoxy-D-glucose. Int J Oncol 35:609615,2009

37. Mohanti BK, Rath GK, Anantha N, et al: Improving cancer radiotherapy with 2-deoxy-D-glucose: Phase I/II clinical trials on human cerebral gliomas. Int J Radiat Oncol Biol Phys 35:103-111, 1996

38. Singh D, Banerji AK, Dwarakanath BS, et al: Optimizing cancer radiotherapy with 2-deoxy-dglucose dose escalation studies in patients with glioblastoma multiforme. Strahlenther Onkol 181 507-514, 2005

39. Chan DA, Sutphin PD, Nguyen $P$, et al: Targeting GLUT1 and the Warburg effect in renal cell carcinoma by chemical synthetic lethality. Sci Transl Med 3:94ra70, 2011

40. Kennedy KM, Dewhirst MW: Tumor metabolism of lactate: The influence and therapeutic potential for MCT and CD147 regulation. Future Oncol 6:127-148, 2010

41. Halestrap AP, Wilson MC: The monocarboxylate transporter family: Role and regulation. IUBMB Life 64:109-119, 2012

42. Sonveaux P, Vegran F, Schroeder T, et al: Targeting lactate-fueled respiration selectively kills hypoxic tumor cells in mice. J Clin Invest 118:3930-3942, 2008

43. Miranda-Goncalves V, Honavar M, Pinheiro C, et al: Monocarboxylate transporters (MCTs) in gliomas: Expression and exploitation as therapeutic targets. Neuro Oncol 15:172-188, 2013

44. Krause DS, Van Etten RA: Tyrosine kinases as targets for cancer therapy. N Engl J Med 353:172 187, 2005

45. Verellen D, De Ridder M, Linthout $N$, et al: Innovations in image-guided radiotherapy. Nat Rev Cancer 7:949-960, 2007

46. Eisbruch $A$ : Intensity-modulated radiation therapy in the treatment of head and neck cancer: Nature clinical practice. Oncology 2:34-39, 2005

47. Kastan MB: DNA damage responses: Mechanisms and roles in human disease: 2007 G.H.A. Clowes Memorial Award Lecture. Mol Cancer Res 6:517-524, 2008

48. Guo K, Shelat AA, Guy RK, et al: Development of a cell-based, high-throughput screening assay for ATM kinase inhibitors. J Biomol Screening 19:538546, 2014

49. Chan DA, Giaccia AJ: Harnessing synthetic lethal interactions in anticancer drug discovery. Nat Rev Drug Discov 10:351-364, 2011
50. Ashworth A: A synthetic lethal therapeutic approach: Poly(ADP) ribose polymerase inhibitors for the treatment of cancers deficient in DNA double-strand break repair. J Clin Oncol 26:37853790, 2008

51. Bindra RS, Schaffer PJ, Meng A, et al: Downregulation of Rad51 and decreased homologous recombination in hypoxic cancer cells. Mol Cell Biol 24:8504-8518, 2004

52. Chan N, Koritzinsky M, Zhao $\mathrm{H}$, et al: Chronic hypoxia decreases synthesis of homologous recombination proteins to offset chemoresistance and radioresistance. Cancer Res 68:605-614, 2008

53. Chan N, Bristow RG: "Contextual" synthetic lethality and/or loss of heterozygosity: Tumor hypoxia and modification of DNA repair. Clin Cancer Res 16:4553-4560, 2010

54. Higgins GS, Prevo R, Lee YF, et al: A small interfering RNA screen of genes involved in DNA repair identifies tumor-specific radiosensitization by POLQ knockdown. Cancer Res 70:2984-2993, 2010

55. Spencer CM, Goa KL: Amifostine: A review of its pharmacodynamic and pharmacokinetic properties, and therapeutic potential as a radioprotector and cytotoxic chemoprotector. Drugs 50:1001-1031, 1995

56. Weiss JF, Landauer MR: Protection against ionizing radiation by antioxidant nutrients and phytochemicals. Toxicology 189:1-20, 2003

57. Komarov PG, Komarova EA, Kondratov RV, et al: A chemical inhibitor of p53 that protects mice from the side effects of cancer therapy. Science 285:1733-1737, 1999

58. Kirsch DG, Santiago PM, di Tomaso E, et al: p53 controls radiation-induced gastrointestinal syndrome in mice independent of apoptosis. Science 327:593-596, 2010

59. Burdelya LG, Krivokrysenko VI, Tallant TC, et al: An agonist of toll-like receptor 5 has radioprotective activity in mouse and primate models. Science 320:226-230, 2008

60. Paris F, Fuks Z, Kang A, et al: Endothelial apoptosis as the primary lesion initiating intestinal radiation damage in mice. Science 293:293-297, 2001

61. Taniguchi CM, Miao YM, Diep AN, et al: PHD inhibition mitigates and protects against radiationinduced gastrointestinal toxicity via HIF2. Sci Transl Med 6:236-264, 2014

62. Yan L, Colandrea VJ, Hale JJ: Prolyl hydroxylase domain-containing protein inhibitors as stabilizers of hypoxia-inducible factor: Small molecule- based therapeutics for anemia. Expert Opin Ther Pat 20:1219-1245, 2010

63. Salama JK, Milano MT: Radical irradiation of extracranial oligometastases. J Clin Oncol 32:29022912, 2014

64. Benderitter M, Caviggioli F, Chapel A, et al: Stem cell therapies for the treatment of radiationinduced normal tissue side effects. Antioxid Redox Signal 21:338-355, 2014

65. Guha C, Sharma A, Gupta S, et al: Amelioration of radiation-induced liver damage in partially hepatectomized rats by hepatocyte transplantation. Cancer Res 59:5871-5874, 1999

66. Krause $P$, Wolff $H A$, Rave-Frank $M$, et al: Fractionated external beam radiotherapy as a suitable preparative regimen for hepatocyte transplantation after partial hepatectomy. Int J Radiat Oncol Biol Phys 80:1214-1219, 2011

67. Hughes RD, Mitry RR, Dhawan A: Current status of hepatocyte transplantation. Transplantation 93:342-347, 2012

68. Burnette B, Weichselbaum RR: Radiation as an immune modulator. Semin Radiat Oncol 23:273280, 2013

69. Sharma $P$, Wagner $K$, Wolchok JD, et al: Novel cancer immunotherapy agents with survival benefit: Recent successes and next steps. Nat Rev Cancer 11:805-812, 2011

70. Pardoll DM: The blockade of immune checkpoints in cancer immunotherapy. Nat Rev Cancer 12:252-264, 2012

71. Postow MA, Callahan MK, Barker CA, et al: Immunologic correlates of the abscopal effect in a patient with melanoma. N Engl J Med 366:925-931, 2012

72. Hiniker SM, Chen DS, Knox SJ: Abscopal effect in a patient with melanoma. $\mathrm{N}$ Engl $\mathrm{J}$ Med 366:2035, 2012

73. Stamell EF, Wolchok JD, Gnjatic S, et al: The abscopal effect associated with a systemic antimelanoma immune response. Int J Radiat Oncol Biol Phys 85:293-295, 2013

74. Seung SK, Curti BD, Crittenden $M$, et al: Phase 1 study of stereotactic body radiotherapy and interleukin-2: Tumor and immunological responses. Sci Transl Med 4:137ra74, 2012

75. Formenti SC, Demaria S: Combining radiotherapy and cancer immunotherapy: A paradigm shift. J Natl Cancer Inst 105:256-265, 2013 
AUTHORS' DISCLOSURES OF POTENTIAL CONFLICTS OF INTEREST MOLECULAR RADIOBIOLOGY: THE STATE OF THE ART

Molecular Radiobiology: The State of the Art

The following represents disclosure information provided by authors of this manuscript. All relationships are considered compensated. Relationships are self-held unless noted. I = Immediate Family Member, Inst $=$ My Institution. For a detailed description of the disclosure categories, or for more information about ASCO's conflict of interest policy, please refer to the Author Disclosure Declaration and the Disclosures of Potential Conflicts of Interest section in Information for Contributors.

Amato J. Giaccia

Stock or Other Ownership: Proacta, Ruga, FibroGen (Inst)

Honoraria: Cerulean Pharma (Inst)
Consulting or Advisory Role: Proacta, Ruga, FibroGen,

Cerulean Pharma (Inst) 